
\section{$\zeta$}

Additional material is published online only. To view please visit the journal online (http://dx.doi.org/10.1136/ thoraxinl-2014-205513)

Department of Respiratory Medicine, Glenfield Hospital, Leicester, UK

\section{Correspondence to}

Dr Yusuf Vali, Department of Respiratory Medicine, Glenfield Hospital, Leicester LE3 9QP, UK; vali.yusuf@gmail.com

Received 31 March 2014 Accepted 1 April 2014 Published Online First 24 April 2014
CrossMark

To cite: Vali Y, Ladwa R, Bailie $\mathrm{E}$, et al. Thorax 2015;70:291-293.

\title{
Investigating and managing suspected pulmonary embolism in an outpatient setting: the Leicester experience
}

\author{
Yusuf Vali, Rahul Ladwa, Elaine Bailie, Jonathan Bennett, Catherine Free
}

\section{ABSTRACT \\ Having established an ambulatory service for patients with suspected and proven PE, we reviewed our outcomes. All patients referred from June 2010 to May 2012 were analysed. Of 971 patients referred, 905 underwent complete assessment (66 admitted as ineligible). 754 (77.7\%) patients were discharged and required no follow-up. $96(10.6 \%)$ patients had PE confirmed of whom 70 (72.9\%) were managed as outpatients. 14 (1.6\%) patients have died since attending the clinic; no death was related to PE. This audit shows that ambulatory investigation and management of selected low risk patients with suspected PE is safe and reduces hospital admissions.}

\section{INTRODUCTION}

PE is a major cause of admission to hospital. Its symptoms are neither specific nor sensitive and include pleuritic chest pain and dyspnoea. These are common presentations and often have other causes which, if PE was excluded, would not necessitate admission to hospital.

Increasing evidence suggests that it is possible to identify patients with a low risk of death from PE who may be treated safely as outpatients. ${ }^{1-4}$ However, there are little data on ambulatory investigation of PE. We describe our experience of investigating and managing low risk patients with suspected PE in the outpatient setting.

\section{METHODS}

We used the Pulmonary Embolism Severity Index (PESI) combined with other predictive factors to identify a cohort of patients who may be suitable for outpatient assessment and management. ${ }^{2}$ Our aim was to minimise risk to patients and maximise compliance with treatment. Patients were deemed eligible if markers of cardiorespiratory compromise were absent, that is, pulse $<110 \mathrm{bpm}$, systolic blood pressure $>100 \mathrm{~mm} \mathrm{Hg}$, oxygen saturations $>92 \%$ on air, respiratory rate $<30 \mathrm{bpm}$, no history of collapse, normal troponin and no large central clot or features of right heart strain on CT pulmonary angiogram (CTPA). Factors affecting compliance, such as cognitive impairment, lack of transport and access to telephone, precluded patients. Patients with a high bleeding risk were excluded alongside those with a coexisting problem requiring hospital admission (see online supplementary table S1).

Patients with suspected PE who met all the eligibility criteria were referred to the service at
Glenfield Hospital, Leicester, from June 2010. We collected and analysed data from the first 2 years. Assessment was performed by a specialist nurse using a standard protocol. Routine blood tests including a latex agglutination D-dimer test, chest radiograph and ECG were performed on all patients. British Thoracic Society (BTS) clinical probability of PE was documented in all patients. In patients with clinical features compatible with PE this is calculated by assessing (a) the absence of another reasonable clinical explanation and (b) the presence of a major risk factor. In the presence of both (a) and (b), the probability is high; the presence of only (a) or (b) gives an intermediate probability; and the absence of (a) and (b) gives a low probability. $^{5}$

For those patients with low or intermediate pretest probability of PE and negative D-dimers, an alternative diagnosis was sought and they were discharged back to primary care. Patients with positive D-dimers or a high pretest probability of PE underwent imaging. Those with no previous cardiorespiratory diagnosis and a normal chest radiograph had ventilation perfusion (VQ) scans. All others had CTPA (algorithm shown in online supplementary figure S1).

Scans were performed on the same day, but where this was not possible patients were discharged on a treatment dose of low molecular weight heparin to return for imaging the following working day.

Data were collected prospectively on a database. Missing information was completed from results and imaging systems.

\section{RESULTS}

Patient characteristics

Figure 1 shows the study profile. During the study period, 971 patients were referred, 66 of whom were admitted prior to complete assessment as they were unsuitable for outpatient management. In all, 905 patients were assessed; median (range) age of 49 (18-92) years, and 614 (67.8\%) were women.

\section{Assessment for PE}

Clinical probability of PE was low in 569 (62.9\%), intermediate in $242(26.7 \%)$ and high in 91 patients $(10.1 \%)$. For those with a low or intermediate probability, the $\mathrm{D}$-dimer was positive in 499 (61.3\%). VQ scans and CTPAs were performed in $562(63.1 \%)$ patients. A total of 52 patients were clinically judged as not having a 


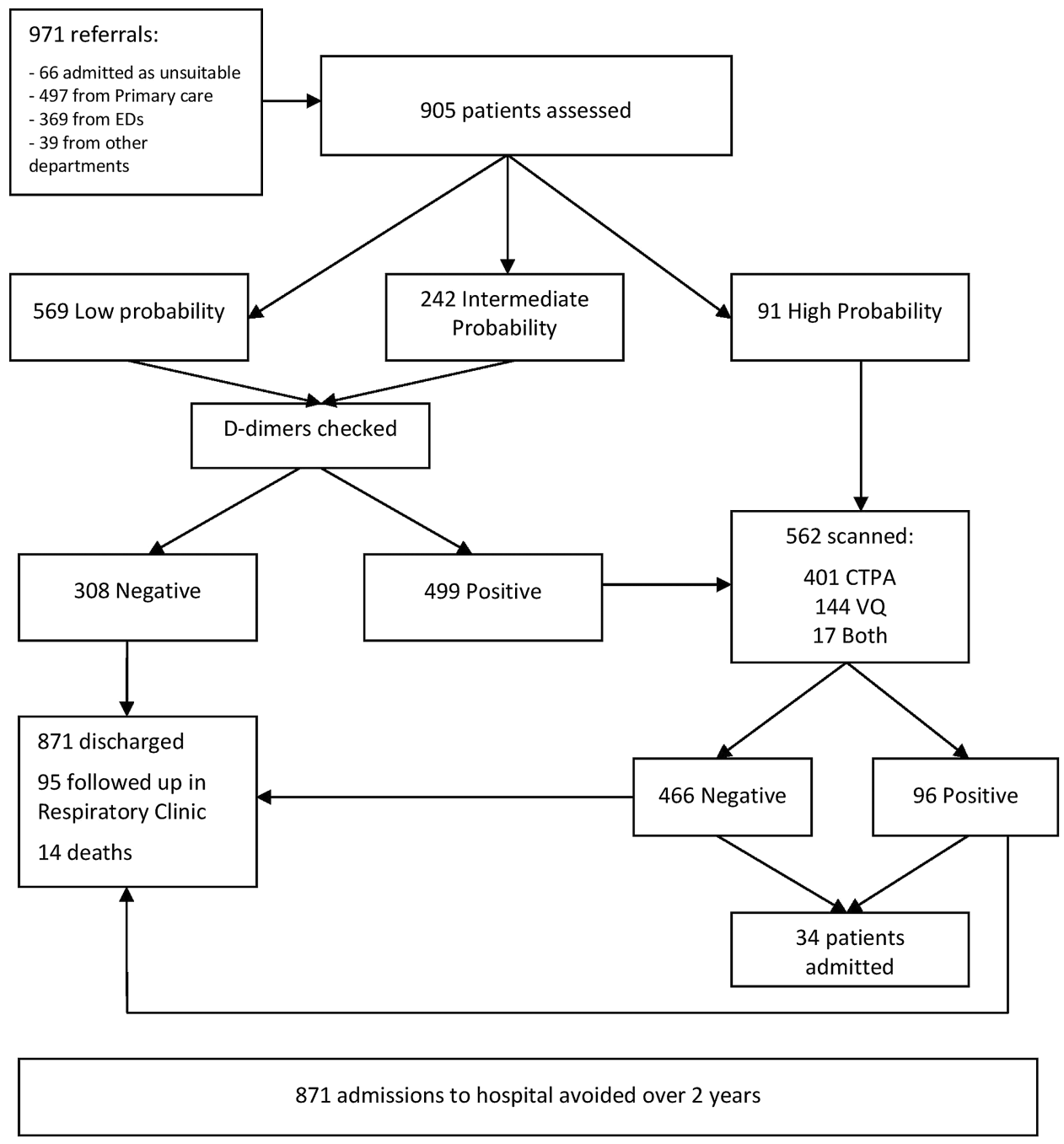

Figure 1 Study profile.

reasonable possibility of PE in the presence of a clear alternative diagnosis and hence further imaging was not required. Overall, 20 patients with a negative D-dimer assay whose BTS clinical probability score was low or intermediate were offered further imaging on the basis of clinical judgement. The remaining 288 (32.7\%) patients with negative D-dimers required no further imaging and an alternative diagnosis was sought.

The median (range) time to imaging was $0(0-5)$ days, with $353(62.8 \%)$ patients having their scan on the same day, 147 (26.2\%) waiting until the next day, 19 (3.4\%) 2 days, 31 (5.5\%) 3 days, $10(1.8 \%) 4$ days and $2(0.4 \%) 5$ days.

\section{Pulmonary embolism}

PE was confirmed in 96 patients (42; 43.7\% women), median age 59.5 years. These patients constituted $17.1 \%$ of those scanned and $10.6 \%$ of the total number seen.

\section{Admissions from clinic}

Of the $100(10.3 \%)$ patients admitted (66 prior to complete assessment) from the clinic, 69 were unsuitable for outpatient assessment (27 had another condition requiring admission, 19 had cardiorespiratory instability, 10 had renal impairment and 13 miscellaneous), 24 had a high risk PE (saddle embolus, right heart strain on CT or raised troponin) and seven were admitted briefly pending results of imaging which were not available by the end of the clinic's working day.

A total of $70(72.9 \%)$ patients with PE had outpatient management. In all, 754 (77.7\%) patients referred with suspected $\mathrm{PE}$ were discharged from clinic and required no follow-up.

\section{Health economics}

Traditional assessment and management of suspected PE will usually result in an inpatient stay. Previous pilot work suggested a mean length of stay (LoS) of 1.59 days and thus deflecting 435.5 admissions per year resulted in 692 saved bed days per year for our catchment population of one million. Patients with confirmed PE have a mean LoS of 6 days. $^{3}$ Our estimated cost savings ( $£ 250$ per bed day) are $£ 173000$ per annum.

\section{Deaths}

As at October 2012, there were $14(1.6 \%)$ deaths in patients who had attended the clinic (median age 76). There were two deaths between days 0 and 30; one between days 31 and 60 ; two between days 61 and 90; and nine deaths beyond this period. No deaths were related to PE or outpatient management of suspected PE. Of these 14 patients, only two had a diagnosis of PE; both died from cancer, one at day 30 and the other beyond 90 days. 


\section{DISCUSSION}

This study demonstrates that our pragmatic approach to the ambulatory assessment and management of selected low risk patients with possible or proven PE is safe, efficient and cost effective.

There is increasing evidence in the literature that patients who are at low risk of death from PE can be identified and treated successfully as an outpatient. The most extensively validated clinical scoring system is the PESI. ${ }^{2}$ It comprises 11 scored domains. Patients with surrogate clinical markers of right heart strain score highest on the PESI, that is, pulse $>110$, systolic BP $<100 \mathrm{~mm} \mathrm{Hg}$, oxygen saturations $<90 \%$, respiratory rate $>30 \mathrm{bpm}$ or a history of collapse or altered mental status. Thus, we adopted these clinical parameters as markers of higher risk patients who were not suitable for ambulatory management. However, a cut-off for oxygen saturations of $92 \%$ rather than $90 \%$ was selected to maximise patient safety while enabling those suitable to be managed as outpatients. Patients with cancer were included as their risk of death was judged to be due to their condition rather than associated with PE. There were no PE related deaths in our study.

Two recent meta-analyses have demonstrated the safety of outpatient treatment of PE in low risk patients. ${ }^{3}{ }^{4}$ Most studies have only considered the safety of outpatient treatment once PE has been diagnosed. The number of patients potentially available is limited by this approach. We chose to apply the same criteria to all patients with suspected $\mathrm{PE}$, the vast majority of whom did not have PE and in whom admission could be avoided.

One of the only comparable studies examining the suitability of outpatient diagnosis of PE was published in 2006. ${ }^{1}$ Hogg et al prospectively studied 408 emergency admissions with pleuritic chest pain. Of these, $353(86.5 \%)$ were safely investigated as outpatients.

Using our service model, we estimated that we saved 692 bed days per year for our catchment population of one million. If this model is adopted across a population of 64 million in the $\mathrm{UK}$, it is postulated a 44000 bed-day reduction annually will result.

The average forecast income is $£ 921$ for each patient seen in the clinic. The majority of patient episodes (chest pain, lower respiratory tract infection, $\mathrm{PE}$, etc) would receive the 'best practice tariff' for same day emergency care which was introduced in 2012 by the Department of Health to incentivise ambulatory services. Equivalent inpatient episodes are estimated to attract an average of $£ 764$. The main additional cost incurred by running this service is the salary of a fulltime specialist nurse (approximately $£ 45000$ ). There is no saving related to investigations as the same number and type are performed.

In summary, this is the largest study demonstrating the feasibility and safety of investigating and managing suspected PE in an ambulatory setting for selected patients. The modified PESI criteria we use to select patients for outpatient investigation successfully identify a low risk group who do not require inpatient monitoring. Additionally, it illustrates the financial advantages of ambulatory care and the resulting avoided admissions. Our cohort is unlikely to be significantly different to other patient populations of a similar age group and hence our results are likely to be reproducible.

Contributors Concept and design: CF, JB, EB. Acquisition of data: YV, RL, EB. Analysis and interpretation: YV, RL. Drafting the manuscript: YV, CF, JB. Guarantor: CF.

Funding The ambulatory PE service at Glenfield Hospital was established with support from an East Midlands regional innovation fund.

Competing interests None.

Provenance and peer review Not commissioned; externally peer reviewed.

Data sharing statement The data from this audit have not been made available to any external body outside of Glenfield Hospital, Leicester.

\section{REFERENCES}

1 Hogg K, Dawson D, Mackway-Jones K. Outpatient diagnosis of pulmonary embolism: the MIOPED (Manchester Investigation of Pulmonary Embolism Diagnosis) study. Emerg Med J 2006;23:123-7.

2 Aujesky D, Obrosky DS, Stone RA, et al. Derivation and validation of a prognostic model for pulmonary embolism. Am J Respir Crit Care Med 2005;172:1041-6.

3 Zondag W, Kooiman J, Klok FA, et al. Outpatient versus inpatient treatment in patients with pulmonary embolism: a meta-analysis. Eur Respir J 2013;42:134-44.

4 Piran S, Le Gal PS, Wells PS, et al. Outpatient treatment of symptomatic pulmonary embolism: A systematic review and meta-analysis. Thromb Res 2013;132:515-19.

5 British Thoracic Society Standards of Care Committee Pulmonary Embolism Guideline Development Group. British Thoracic Society guidelines for the management of suspected acute pulmonary embolism. Thorax 2003;58:470-84. 